\title{
D-Allele of ACE Polymorphism is Associated With Increased Magnitude of QT Dispersion Prolongation in Elderly Chinese 4-Year Follow-up Study
}

\author{
Tsung-Hsien Lin, MD*,†; Herng-Chia Chiu, PhD ${ }^{\dagger}$; Ho-Ming Su, MD*; \\ Suh-Hang Hank Juo, MD*; Ya-Ting Lee, MD**; Wen-Chol Voon, MD*; \\ Wen-Ter Lai, MD*; Sheng-Hsiung Sheu, MD*,†
}

\begin{abstract}
Background There has not been a longitudinal investigation of the influence of angiotensin-converting enzyme (ACE) insertion/deletion and angiotensinogen (AGT) M235T gene polymorphisms on repolarization parameters, such as QT dispersion (QTd) and the peak and the end of the T-wave interval (Tpe).

Methods and Results Electrocardiographys were recorded from 106 elderly Chinese at baseline, and $2^{\text {nd }}$ and $4^{\text {th }}$ year follow-up. The corrected QT (QTc), QTd, QTc dispersion (QTcd) and Tpe were manually calculated. Average age was $72.7 \pm 4.1$ years (range $62-81$ ). QTd, QTcd and Tpe were significantly prolonged (all $\mathrm{p}<0.001$ at the $2^{\text {nd }}$ and $4^{\text {th }}$ year). At the $4^{\text {th }}$ year the magnitude of the QTd prolongation, but not Tpe, was significantly higher in subjects carrying the ACE D allele than non-D-allele carriers ( $\mathrm{p}=0.001)$, as was QTcd $(\mathrm{p}=0.002)$. This association was still significant in the multivariate analyses ( $\mathrm{p}<0.001$ and $\mathrm{p}=0.001$ for QTc and QTed, respectively). No significant correlation was found between repolarization parameters and AGT genotype.

Conclusions This longitudinal study shows that the ageing process is associated with prolongation of QTd, QTcd and Tpe after 4 years follow-up. The elderly Chinese subjects with the ACE D-allele had greater prolongation QTd and QTed. (Circ J 2007; 71: 39-45)
\end{abstract}

Key Words: Ageing; Angiotensin-converting enzyme; Polymorphism; QT dispersion; Repolarization

$\mathbf{T}$ he frequency of arrhythmia increases with age, probably because of age-associated degenerative changes in the conduction system, as well as myocardial fibrosis! Ventricular repolarization abnormality plays an important role in the occurrence of arrhythmia. The QT interval and QT dispersion (QTd), 2 of the repolarization parameters, are prolonged with increasing age, which may contribute to an increased risk of ventricular arrhythmias and cardiac mortality in elderly patients? Several large prospective studies have assessed the predictive value of QTd and corrected QT interval (QTc) dispersion (QTcd) for cardiac and all-cause mortality,3,4 and their results suggest that long QTd and QTcd are associated with cardiovascular morbidity and mortality. More recently, transmural dispersion of repolarization (TDR) has been evaluated by measuring the interval between the peak and the end of the $\mathrm{T}$ wave (Tpe), as well as the Tpe interval divided by the QT interval (Tpe/QT) ratio, creating 2 new repolarization parameters, 6 These increased TDR-related parameters were

(Received May 22, 2006; revised manuscript received October 10, 2006; accepted November 1, 2006)

*Division of Cardiology, Department of Internal Medicine, **Division of Nephrology, Department of Pediatrics, Kaohsiung Medical University Chung-Ho Memorial Hospital, Graduate Institute of Medicine, ${ }^{\dagger}$ Public Health and Medical Genetics, Kaohsiung Medical University, Kaohsiung, Taiwan

Mailing address: Sheng-Hsiung Sheu, MD, Division of Cardiology, Department of Internal Medicine, Kaohsiung Medical University Chung-Ho Memorial Hospital, 100 Shi-Chuan 1st Road, Kaohsiung. 80708, Taiwan, ROC. E-mail: Sheush@kmu.edu.tw found to be associated with the occurrence of arrhythmias, such as torsades de pointes in long QT syndrome6 Furthermore, a cross-sectional study showed that TDR parameters increased with age? Therefore, it is mandatory to determine the risk factors in the elderly that may predispose to prolongation of these repolarization parameters.

The renin-angiotensin system (RAS) is involved in many cardiovascular diseases. Carriers of the D-allele of angiotensin-converting enzyme (ACE) insertion/deletion (I/D) polymorphism display elevated serum and cardiac ACE activity and thus may have higher RAS system activation.,9 Therefore, the D-allele carriers are exposed to a higher level of angiotensin II than non-D allele carriers. Compelling studies report that the ACE D-allele is associated with myocardial infarction, sudden death and malignant ventricular arrhythmia! ${ }^{10-12}$ Furthermore, the D-allele has been associated with QTd in hypertensive subjects or survivors of myocardial infarction!13,14 Angiotensinogen (AGT) M235T polymorphism is associated with plasma AGT levels that increase stepwise according to the number of $\mathrm{T}$ alleles present $\mathrm{I}^{15}$ The $\mathrm{T}$ allele has very different distributions in Asians (70-73\%) and Caucasians (10-24\%)! 16,17 One study showed that homozygosity for the $\mathrm{T}$ allele was associated with increased risk of cardiovascular diseases, such as hypertension, coronary artery disease and myocardial infarction $!^{15}$

To our knowledge, the influence of gene polymorphisms on QTd and TDR-related parameters has not been reported in a prospective cohort. We therefore conducted a prospective study to investigate several physiological parameters 


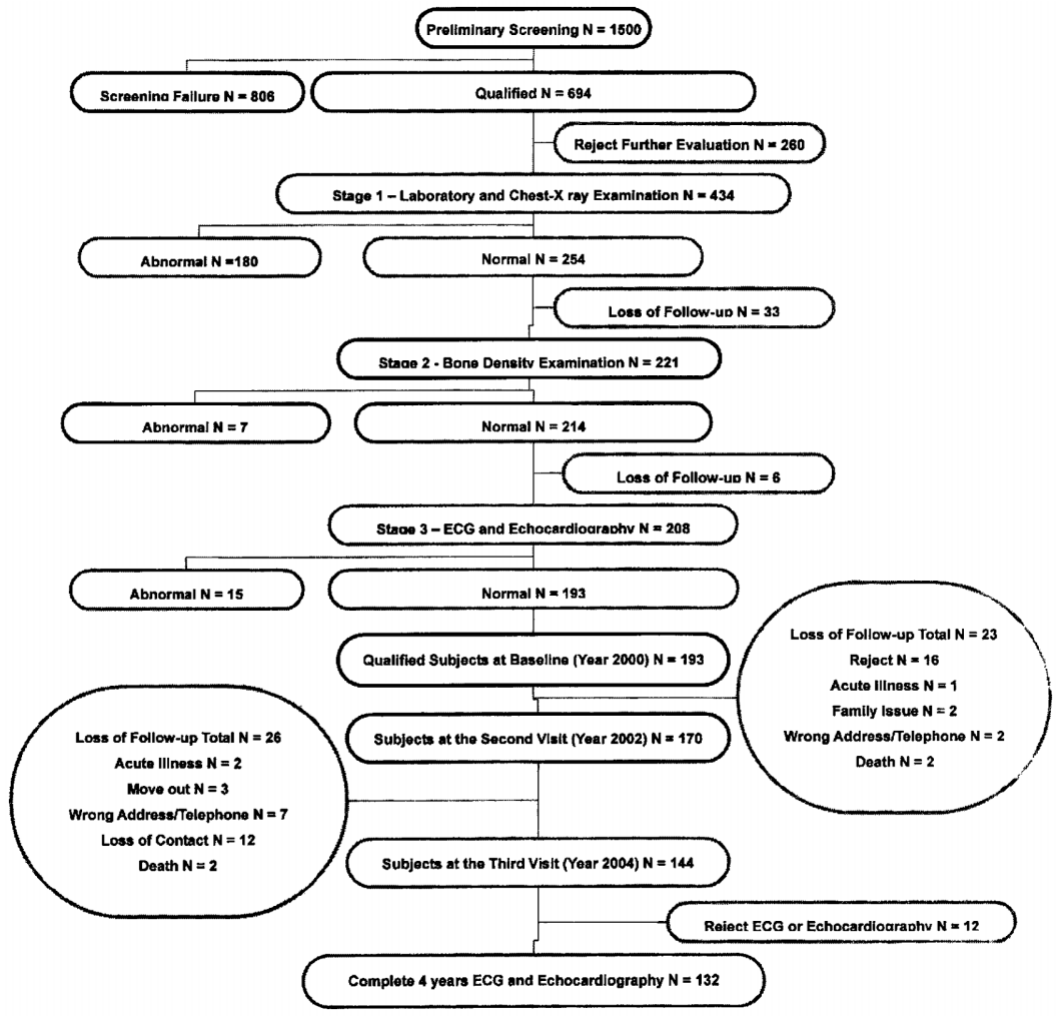

Fig 1. Trial profile. ECG, electrocardiography.

in an elderly Chinese population living in Taiwan. In the present study, we proposed testing for the relationship between the magnitude of QTd, QTed, Tpe and Tpe/QT prolongation and 2 genetic polymorphisms: ACE I/D and AGT M235T.

\section{Methods}

\section{Patients}

The subjects were from a community-based longitudinal cohort study (Gerontology Research Laboratory: The Longitudinal Study of Aging) that was designed to discover changes in several physiological functions and structures with aging in healthy elderly subjects. The working group was first initiated in 2000 and included several co-investigator physicians in the areas of chest medicine, orthopedics and cardiology. Each co-investigator physician was responsible for 1 specific examination of the elderly participants. Each participant had to be free of disease at baseline in order to be recruited into the normal aging group. Participants were invited from several senior activity centers in Kaohsiung city located in southern Taiwan. Baseline examinations were performed from October 1, 2000 to March 31,2001 .

The screening process included 3 stages. Fig 1 shows the number of participants at each stage of screening or examination. There were 1,500 potential candidates at the beginning. After being interviewed by senior nurses using a constructed questionnaire, 694 qualified for the study. However, only 434 elderly candidates were willing to visit Kaohsiung Medical University (KMU) hospital for biochemical tests and physiological examination as the first stage. The results of the laboratory testing and chest radiography were carefully examined by 2 senior physicians and 254 cases qualified as healthy elderly and 180 did not meet the criteria. In the second stage, 221 of the 254 subjects continued to participate in the study for bone density (BMD) evaluation. The BMD evaluation excluded 7 unqualified subjects and 6 subjects were lost to follow-up. Finally, only 208 subjects participated in the third stage examinations of electrocardiography (ECG) and echocardiography. Of them, 193 cases had normal ECG and echocardiographic data and were enrolled as the cardiology study population; the remaining 15 cases did not meet the criteria. Inclusion criteria for healthy elderly subjects in the cardiology group were: (1) age $\geq 60$ years old; (2) active, with no limitations in daily life; (3) no history of previously diagnosed diabetes, hypertension, ischemic heart disease or cerebrovascular accident, (4) no significant ECG or echocardiographic abnormality, including atrial fibrillation and myocardial infarction, and (5) not on any chronic medications. This study was approved by the Institutional Review Board, KMU Hospital. All enrolled patients gave informed consent.

Blood pressure was taken after resting comfortably for $10 \mathrm{~min}$. A computerized automatic mercury-sphygmomanometer (CH-5000, Citizen, Tokyo, Japan) was used on the right arm while participants were seated. The reading was repeated and the mean of 2 readings was used as baseline data. Hypertension was defined as a systolic blood pressure $\geq 140 \mathrm{mmHg}$ or a diastolic blood pressure $\geq 90 \mathrm{mmHg}$. Body weight and height were measured and body mass index (BMI) was calculated. All of the measurements were repeated every 2 years. The examinations were usually scheduled in the same month as the first visit to ensure the same interval between follow-up visits.

\section{$E C G$}

A standard 12-lead resting ECG was recorded at a paper speed of $25 \mathrm{~mm} / \mathrm{s}$ and a gain of $10 \mathrm{~mm} / \mathrm{mV}$ at baseline, and 
years 2 and 4. To reduce the impact of the circadian variation of QTd, the ECG examinations were completed in the morning $(08.00$ to $10.00 \mathrm{~h})$. All ECGs, with those at the same observational stage as a pack, were manually analyzed by 1 experienced cardiologist (H.S.) who was unaware of the clinical data. Subjects with complete bundle branch block, 2nd or 3rd degree atrioventricular block, or less than 9 measurable leads on the ECG recording were excluded.

The QT was defined as the interval between the QRS onset and the end of the T wave, at the point where the isoelectric line intersected a tangential line drawn on the maximal downslope of the positive T wave! ${ }^{18}$ QTc according to the Bazett's formula. QT and QTcd were calculated as the maximal QT and QTc minus the minimal QT and QTc interval, respectively. The interval between Tpe was measured using the V5 lead (Fig 2)! ${ }^{19}$ Tpe/QT was calculated as the relative Tpe/QT. All of the measurements were performed as the mean of approximately 3 consecutive beats.

The prolongation of QTd was defined as QTd at years 2 and 4 minus QTd at baseline. The prolongation of each of QTed, Tpe and Tpe/QT was calculated the same way. The left ventricle (LV) was measured by echocardiography and LV mass was calculated according to the formula of Devereux and Reichek ${ }^{20}$ then divided by body surface area to give the LV mass index (LVMI) used in the present study.

\section{Extraction and Amplification of Genomic DNA}

In accordance with standard methodology, genomic DNA was extracted from peripheral blood with a blood DNA kit (Puregene Gentra System, Minneapolis, MN, USA). Genomic DNA was suspended in $10 \mathrm{mmol} / \mathrm{L}$ Tris $\mathrm{HCl}$ and $1 \mathrm{mmol} / \mathrm{L}$ EDTA $\mathrm{pH}$ 8.0, and the concentrations of DNA were measured by spectrophotometry.

The ACE I/D and AGT M235 gene polymorphisms were analyzed by polymerase chain reaction as previously described in detail. ${ }^{21,22}$

\section{Statistical Analysis}

All data are expressed as mean \pm standard deviation. The paired t-test was used to evaluate the change in continuous variables during the 4-year follow-up. The genotypic distribution was tested by Hardy-Weinberg equilibrium. We used the ANOVA to test for overall significant differences in the baseline characteristics among the different genotypes. Pair-wise comparisons between any 2 of the 3 genotypes were tested by using the Scheffé's method. We then used multiple linear regression analysis to test for the association between gene and phenotypes with adjustment for other covariates. Our independent covariates included sex, age, BMI, the presence of hypertension and LVMI at baseline. All tests were 2-sided, and the level of significance was established as $p<0.05$. The Statistical Package for the Social Sciences (SPSS) 11.0 for Windows (SPSS Inc, Chicago, IL, USA) was used for statistical analysis.

\section{Results}

\section{Clinical Characteristics}

Of the 193 subjects enrolled initially, 132 completed the year 4 examinations of ECG and echocardiography (Fig 1). One-hundred and fifteen subjects provided blood for genetic analysis but 9 of them were excluded because of uninterpretable ECG or restriction digest results for genotypes. A final total of 106 subjects were included in the current

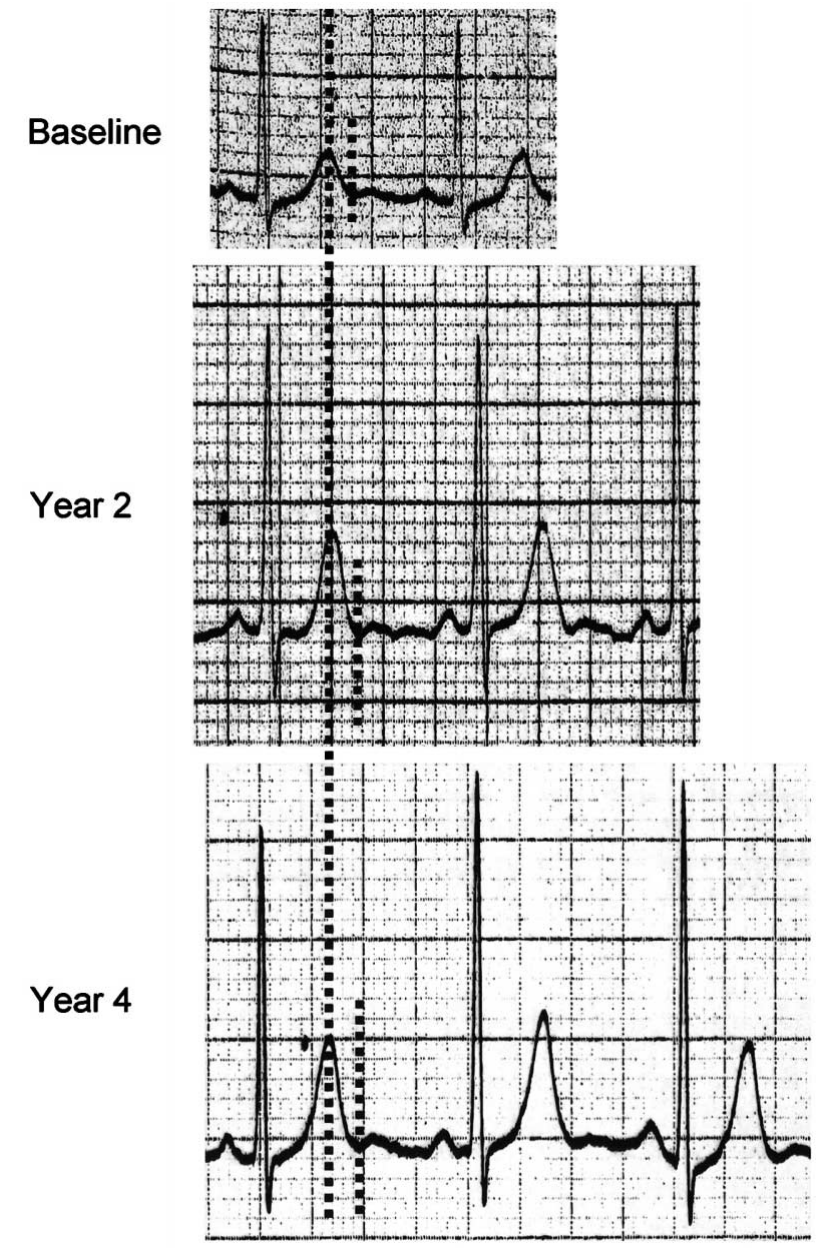

Fig 2. Changes in the peak and the end of the $\mathrm{T}$ wave interval (Tpe). The dotted lines show the peak of the $\mathrm{T}$ wave and the end of the $\mathrm{T}$ wave at the baseline and $2^{\text {nd }}$ and $4^{\text {th }}$ year. The voltage at the baseline is halved. The lead is V5.

study. Table 1 summarizes the demographic information at baseline and the $2^{\text {nd }}$ and $4^{\text {th }}$ years. The subjects included 81 men and 25 women. Ages at enrollment ranged from 62 to 81 years old (mean 72.7 \pm 4.1 ). At the end of the 4-year follow-up, weight and height were significantly reduced. There was an increase in the LVMI $(97.9 \pm 25.4$ to $105.9 \pm$ $29.7 \mathrm{~g} / \mathrm{m}^{2}, \mathrm{p}=0.001$ ) at the $3 \mathrm{rd}$ examination (Table 1 ). No significant arrhythmia was found during the follow-up period. There were 2 and 1 subjects suffering from asymptomatic atrial and ventricular premature beats, respectively, in the $2^{\text {nd }}$ year. The ECG showed atrial and ventricular premature beat in another 2 and 1 participants, respectively, in the $4^{\text {th }}$ year.

There was no statistical significance in baseline characteristics, including sex (male, 75 vs 84\%, $\mathrm{p}=0.159$ ), age $(72.7 \pm 4.4$ vs $73.7 \pm 3.2, \mathrm{p}=0.087)$, BMI $(23.3 \pm 3.1$ vs $23.0 \pm$ $\left.3.1 \mathrm{~kg} / \mathrm{m}^{2}, \quad \mathrm{p}=0.453\right)$, LVMI $(162.4 \pm 45.3$ vs $165.9 \pm$ $\left.51.3 \mathrm{gm} / \mathrm{m}^{2}, \mathrm{p}=0.621\right)$, systolic $(125.1 \pm 14.1$ vs $127.5 \pm$ $13.2 \mathrm{mmHg}, \mathrm{p}=0.230)$ and diastolic $(71.9 \pm 8.8$ vs $73.2 \pm$ $9.6 \mathrm{mmHg}, \mathrm{p}=0.348$ ) blood pressures between the subjects who did or did not complete the 4-year follow-up.

\section{ACE I/D and AGT M235T Gene Polymorphism}

Among the 106 study subjects, 7 (6.6\%), 42 (39.6\%) and $57(53.8 \%)$ had the ACE DD, DI and II genotypes, respec- 
Table 1 Patients' Characteristics and QT Variables

\begin{tabular}{|c|c|c|c|c|c|}
\hline & \multirow{2}{*}{ Baseline } & \multirow{2}{*}{$2^{\text {nd }}$ year } & \multirow{2}{*}{$4^{\text {th }}$ year } & \multicolumn{2}{|c|}{$p$ value } \\
\hline & & & & Year 2-baseline & Year 4-baseline \\
\hline No. & & 106 & & & \\
\hline Male no. (\%) & & $81(76.4)$ & & & \\
\hline Age (years) & $72.7 \pm 4.1$ & $74.7 \pm 4.1$ & $76.7 \pm 4.1$ & & \\
\hline Weight $(\mathrm{kg})$ & $61.3 \pm 8.7$ & $60.3 \pm 8.8$ & $60.3 \pm 9.5$ & $<0.001$ & 0.005 \\
\hline Height $(\mathrm{cm})$ & $162.1 \pm 7.1$ & $161.6 \pm 7.0$ & $161.7 \pm 8.2$ & $<0.001$ & $<0.001$ \\
\hline$B M I\left(k g / m^{2}\right)$ & $23.3 \pm 3.0$ & $23.1 \pm 3.1$ & $23.1 \pm 3.3$ & 0.049 & 0.055 \\
\hline HR (beats/min) & $68.9 \pm 9.6$ & $69.0 \pm 9.6$ & $70.2 \pm 11.6$ & 0.783 & 0.177 \\
\hline $\mathrm{SBP}(\mathrm{mmHg})$ & $125.0 \pm 14.2$ & $123.4 \pm 14.1$ & $125.6 \pm 16.1$ & 0.335 & 0.730 \\
\hline$D B P(m m H g)$ & $72.0 \pm 8.8$ & $72.4 \pm 10.9$ & $73.0 \pm 12.7$ & 0.832 & 0.361 \\
\hline $\operatorname{LVMI}\left(\mathrm{gm} / \mathrm{m}^{2}\right)$ & $97.9 \pm 25.4$ & $101.2 \pm 26.0$ & $105.9 \pm 29.7$ & 0.171 & 0.001 \\
\hline$Q T c(m s)$ & $422.4 \pm 20.4$ & $424.1 \pm 21.0$ & $428.9 \pm 28.0$ & 0.300 & 0.005 \\
\hline$Q T d(m s)$ & $33.7 \pm 11.6$ & $38.1 \pm 12.8$ & $43.2 \pm 12.0$ & $<0.001$ & $<0.001$ \\
\hline$Q T c d(m s)$ & $35.8 \pm 12.4$ & $40.7 \pm 13.7$ & $46.5 \pm 13.5$ & $<0.001$ & $<0.001$ \\
\hline Tpe (ms) & $71.6 \pm 13.4$ & $77.6 \pm 11.2$ & $91.9 \pm 12.3$ & $<0.001$ & $<0.001$ \\
\hline Tpe/QT & $0.181 \pm 0.032$ & $0.196 \pm 0.028$ & $0.231 \pm 0.030$ & $<0.001$ & $<0.001$ \\
\hline
\end{tabular}

Data are mean \pm standard deviation.

BMI, body mass index; HR, heart rate; SBP, systolic blood pressure; DBP, diastolic blood pressure; LVMI, left ventricular mass index; QTc, corrected QT interval; QTd, QT dispersion; QTcd, QTc dispersion; Tpe, interval between the peak and the end of the T wave; Tpe/QT, Tpe interval divided by the QT interval.

Table 2 Different ACE Genotypes, Clinical Characteristics and QT Variables

\begin{tabular}{|c|c|c|c|c|}
\hline & \multicolumn{3}{|c|}{ ACE Genotype } & \multirow{2}{*}{$p$ value } \\
\hline & II & $I D$ & $D D$ & \\
\hline No. & 57 & 42 & 7 & \\
\hline Age (years) & $72.2 \pm 4.3$ & $73.2 \pm 3.5$ & $72.4 \pm 5.2$ & 0.487 \\
\hline Weight $(\mathrm{kg})$ & $61.8 \pm 8.9$ & $60.5 \pm 8.8$ & $61.4 \pm 7.7$ & 0.762 \\
\hline Height $(\mathrm{cm})$ & $162.4 \pm 7.4$ & $162.0 \pm 7.5$ & $161.3 \pm 4.3$ & 0.912 \\
\hline$B M I\left(\mathrm{~kg} / \mathrm{m}^{2}\right)$ & $23.5 \pm 3.3$ & $23.0 \pm 2.7$ & $23.6 \pm 2.9$ & 0.738 \\
\hline HR (beats/min) & $70.4 \pm 9.2$ & $66.6 \pm 9.1$ & $70.0 \pm 14.3$ & 0.138 \\
\hline$S B P(m m H g)$ & $123.7 \pm 15.1$ & $126.5 \pm 12.7$ & $126.1 \pm 15.3$ & 0.616 \\
\hline$D B P(m m H g)$ & $72.8 \pm 9.2$ & $70.9 \pm 8.1$ & $72.0 \pm 9.6$ & 0.585 \\
\hline $\operatorname{LVMI}\left(\mathrm{gm} / \mathrm{m}^{2}\right)$ & $97.1 \pm 27.0$ & $97.8 \pm 23.1$ & $101.6 \pm 29.0$ & 0.908 \\
\hline \multicolumn{5}{|l|}{$Q T d(m s)$} \\
\hline Baseline & $35.4 \pm 11.8$ & $32.7 \pm 10.8$ & $25.3 \pm 12.8$ & 0.076 \\
\hline Year 2 & $36.3 \pm 12.1$ & $40.6 \pm 12.6$ & $37.3 \pm 18.7$ & 0.255 \\
\hline Year 4 & $41.5 \pm 11.3$ & $45.6 \pm 11.9$ & $43.4 \pm 17.4$ & 0.233 \\
\hline$\Delta$ Year2-0 & $1.0 \pm 10.4$ & $7.9 \pm 7.7$ & $12.0 \pm 10.3$ & 0.0003 \\
\hline$\Delta$ Year4-0 & $6.1 \pm 9.9$ & $12.9 \pm 10.6$ & $18.1 \pm 14.3$ & 0.001 \\
\hline \multicolumn{5}{|l|}{$Q T c d(m s)$} \\
\hline Baseline & $38.0 \pm 12.5$ & $34.4 \pm 11.6$ & $26.6 \pm 12.3$ & 0.043 \\
\hline Year 2 & $39.1 \pm 13.5$ & $42.8 \pm 12.8$ & $40.7 \pm 20.1$ & 0.410 \\
\hline Year 4 & $44.9 \pm 11.8$ & $49.0 \pm 15.0$ & $45.2 \pm 17.3$ & 0.304 \\
\hline$\Delta$ Year2-0 & $1.0 \pm 11.0$ & $8.4 \pm 8.4$ & $14.1 \pm 12.3$ & 0.0002 \\
\hline$\Delta$ Year4-0 & $6.8 \pm 11.1$ & $14.3 \pm 13.1$ & $18.6 \pm 14.5$ & 0.002 \\
\hline \multicolumn{5}{|l|}{ Tpe (ms) } \\
\hline Baseline & $70.6 \pm 14.2$ & $72.6 \pm 12.7$ & $73.8 \pm 11.8$ & 0.697 \\
\hline Year 2 & $77.0 \pm 11.4$ & $77.6 \pm 10.8$ & $82.3 \pm 13.4$ & 0.506 \\
\hline Year 4 & $90.6 \pm 12.1$ & $93.1 \pm 12.7$ & $95.7 \pm 11.9$ & 0.420 \\
\hline$\Delta$ Year2-0 & $6.4 \pm 11.2$ & $5.0 \pm 11.4$ & $8.5 \pm 7.9$ & 0.687 \\
\hline$\Delta$ Year4-0 & $20.0 \pm 12.6$ & $20.5 \pm 15.1$ & $21.9 \pm 15.7$ & 0.934 \\
\hline \multicolumn{5}{|l|}{ Tpe/QT } \\
\hline Baseline & $0.180 \pm 0.033$ & $0.181 \pm 0.033$ & $0.188 \pm 0.022$ & 0.809 \\
\hline Year 2 & $0.196 \pm 0.028$ & $0.194 \pm 0.028$ & $0.204 \pm 0.025$ & 0.714 \\
\hline Year 4 & $0.230 \pm 0.029$ & $0.232 \pm 0.032$ & $0.235 \pm 0.029$ & 0.887 \\
\hline$\Delta$ Year2-0 & $0.016 \pm 0.027$ & $0.013 \pm 0.029$ & $0.016 \pm 0.028$ & 0.830 \\
\hline$\Delta$ Year4-0 & $0.050 \pm 0.033$ & $0.050 \pm 0.038$ & $0.047 \pm 0.041$ & 0.977 \\
\hline
\end{tabular}

Data are mean \pm standard deviation.

$A C E$, angiotensin converting enzyme; $\triangle$ Year2-0, QT variables prolongation at year 2 minus baseline; $\triangle Y e a r 4-0, Q T$ variables prolongation at year 4 minus baseline. Other abbreviations see in Table 1.

tively; $11(10.4 \%), 30(28.3 \%)$ and $65(61.3 \%)$ had the AGT MM, MT and TT genotypes, respectively. The genotypic distribution was in Hardy-Weinberg equilibrium for both genes. There was no significant difference among the ACE or AGT genotypes regarding baseline age, BMI, systolic and diastolic blood pressures, and LVMI (Tables 2,3).
The ACE genotypes were only associated with QTcd at baseline $(\mathrm{p}=0.043)$ but not with QTcd in years 2 and 4, QTd at baseline and years 2 and 4, or QTc at baseline and years 2 and 4 . The AGT genotypes did not correlate with these variables. Neither ACE nor AGT gene polymorphism correlated with Tpe and Tpe/QT at baseline or years 2 and 4 . 
Table 3 Different AGT Genotypes, Clinical Characteristics and QT Variables

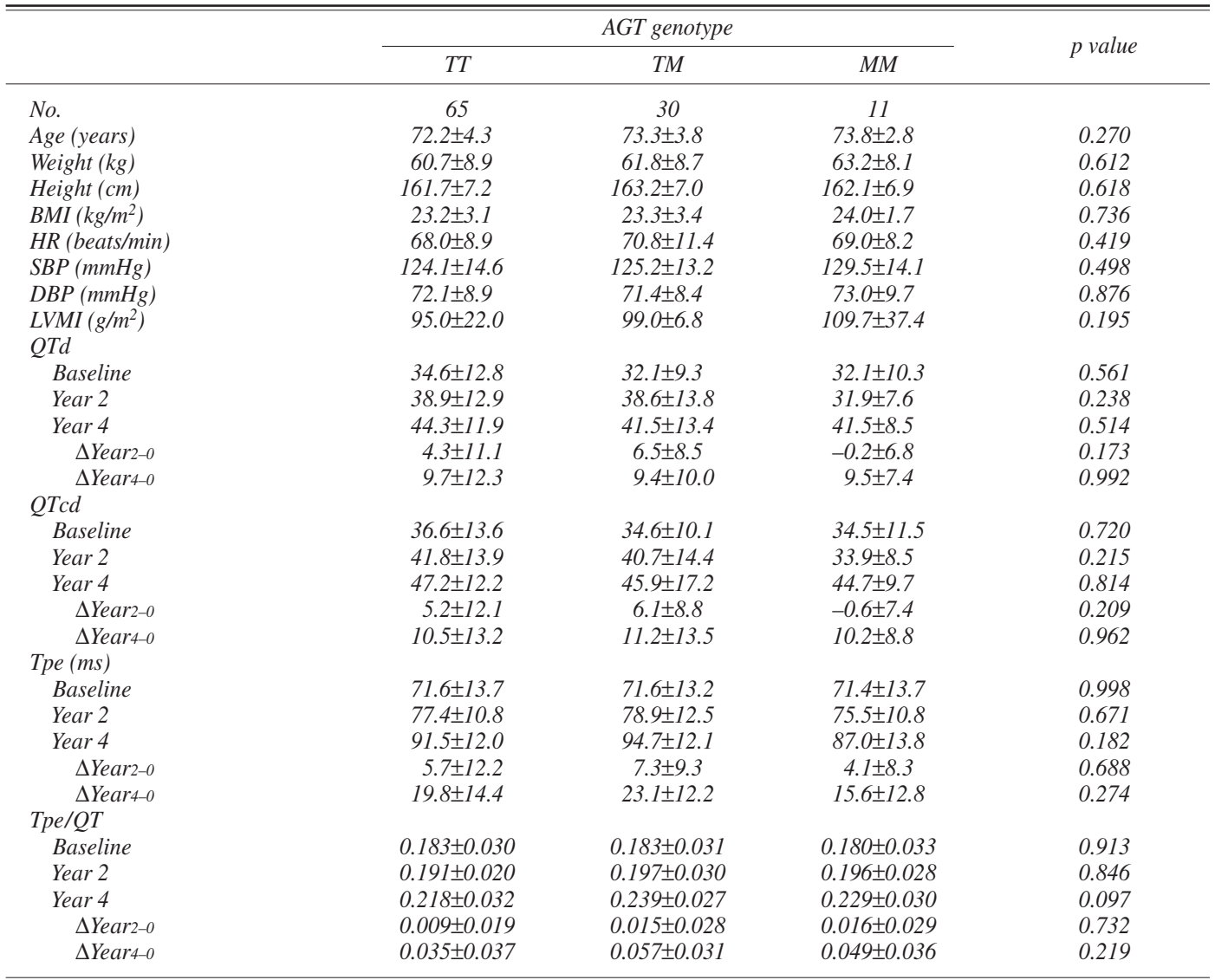

Data are mean \pm standard deviation.

AGT, angiotensinogen. Other abbreviations see in Tables 1,2.

Table 4 Independent Predictors for Magnitude of QTd and QTed Prolongation in Multiple Regression Analysis

\begin{tabular}{lrrrrr}
\hline \hline & \multicolumn{2}{c}{ Year 2} & & \multicolumn{2}{c}{ Year 4} \\
\cline { 2 - 3 } \cline { 5 - 5 } & Coefficient & $p$ value & & Coefficient & $p$ value \\
\hline Magnitude of QTd Prolongation & & & & \\
ACE polymorphism & 6.125 & $<0.001$ & & 6.305 & $<0.001$ \\
$\quad$ Sex & -0.133 & 0.954 & & -2.056 & 0.421 \\
Age & 0.077 & 0.757 & & 0.316 & 0.251 \\
BMI & 0.130 & 0.699 & & 0.326 & 0.382 \\
LVMI & 0.045 & 0.676 & & 0.046 & 0.262 \\
Presence of HT & -1.047 & 0.393 & & -0.362 & 0.896 \\
Magnitude of QTcd Prolongation & & & & 0.001 \\
ACE polymorphism & 6.865 & $<0.001$ & & 6.599 & 0.511 \\
Sex & -0.746 & 0.763 & & -1.915 & 0.198 \\
Age & 0.087 & 0.743 & & 0.404 & 0.487 \\
BMI & 0.156 & 0.664 & & 0.295 & 0.063 \\
LVMI & 0.050 & 0.214 & & 0.088 & 0.679 \\
Presence of HT & -1.010 & 0.706 & & -1.308 & \\
\hline
\end{tabular}

ACE, angiotensin-converting enzyme; HT, hypertension. Other abbreviations see in Table 1.

\section{ECG Data}

The QTc interval, QTd, QTcd Tpe and Tpe/QT increased during the follow-up period (Table 1). The increase in both QTd and QTcd at year 2, as well as at year 4, was proportional to the number of $\mathrm{D}$ alleles, which suggests a potential dose effect (Table 2). The magnitude of the QTd prolongation was significantly higher in subjects with the ACE D-allele than in those who were non D-allele carriers at both year $2(\mathrm{p}=0.0003)$ and $4(\mathrm{p}=0.001)$. The prolongation of QTcd between baseline and the follow-up period also demonstrated the same pattern ( $p$ values $=0.0002$ and 0.002 at year 2 and year 4 , respectively). For the AGT polymorphism, we did not find any association between genotypes and QT variables at baseline, or years 2 and 4. The prolongation of the QTd variables was not related to AGT genotypes (Table 3). The Tpe and Tpe/QT increases were not related to either ACE or AGT genotype.

Multivariate linear regression analysis in which ACE polymorphism, gender, age, BMI, the presence of hypertension and LVMI at baseline were included showed that 
the ACE I/D polymorphism was the only significant determinant $(\mathrm{p}<0.001)$ for the magnitude of QTd prolongation at either year 2 or 4 (Table 4$)$. Therefore, only ACE I/D polymorphism $(p \leq 0.001)$ could significantly predict the magnitude of QTcd prolongation at both years 2 and 4 .

\section{Discussion}

The primary finding of this 4-year longitudinal study was that ACE I/D polymorphism was associated with prolongation of QT and QTcd. We have found that the aging process was associated with prolongation of the QTc interval, QTd and QTed in healthy elderly subjects ${ }^{23} \mathrm{We}$ also found that those subjects carrying the ACE D-allele had a higher magnitude of QTd and QTcd prolongation. Furthermore, our data indicate that the $\mathrm{D}$ allele may have a dose effect on the increase of QT and QTcd.

The QT interval prolongs with increasing age, which may partially explain the increased risk of ventricular arrhythmias and cardiac mortality in the elderly population? Several large prospective studies have reported that QTd and QTcd are determinants for cardiac and all-cause mortality 3,4 Previous cross-sectional studies have not been able to reach a conclusion regarding whether age is a risk for QTd2,14,24-27 These inconsistent findings may be related to the limitations of cross-sectional studies. Our prospective study may be less subject to ascertainment bias than cross-sectional studies. In addition, we investigated elderly subjects, who may be more likely to be predisposed to QT parameters alternation during follow-up than young subjects. Therefore, our data are more reliable for evaluating the effect of ageing on the QTc interval and QTd.

Changes in QTd have been shown to follow the spontaneous or induced dynamicity of the pathological process in some cardiac diseases. Several factors have been demonstrated to cause prolongation of QT parameters; for example, age, medications and myocardial ischemia have been reported as risk factors 18,28 Age is strongly related to degenerative changes in the conduction system, as well as to myocardial fibrosis, both of which lead to QT prolongation. In addition, LVMI and LV hypertrophy ( $\mathrm{LVH}$ ) were associated with a change in the QT interval and QTd in previous large cross-sectional study ${ }^{29}$ In the present study, none of the subjects had organic heart disease or was on chronic medications, which would have influenced the QT variables. Our analysis also indicated that age and LVMI were not independent predictors for the magnitude of QTd and QTed prolongation. There must be something else contributing to the changes in these QT variables.

ACE genotypes were reported to be associated with increased QTd in previous cross-sectional studies $!^{13,14}$ Histopathologic evaluation reveals a maladaptive remodeling of the interstitium associated with ageing, resulting in an increase in patchy myocardial fibrosis and cardiac hypertrophy ${ }^{30}$ The ACE D-allele was associated with an elevation of serum propeptide type I C-terminal procollagen concentration, a marker of myocardial fibrosis, possibly leading to myocardial fibrosis and increased QTd ${ }^{29}$ Subjects carrying the ACE D-allele have a higher risk for developing LVH 31 The myocyte hypertrophy may be associated with a significant prolongation of the transmembrane action potential, which provides an explanation for the prolongation of the QT variables with ageing?2 $\mathrm{We}$ have found that the aging process is associated with an increase of the LVMI and changes of cardiac geometry in the healthy elderly. Further- more, the magnitude of the LVMI increase was significantly higher in subjects carrying the ACE D-allele than in the non-D-allele carriers (unpubl. data). Kuznetsova et al have also reported that LVM is related to ACE I/D polymorphism.33 Their data are consistent with the present findings of changes of the QT variables in the different ACE genotypes and partially explain why the ACE D-allele carriers had higher magnitude of QTd and QTcd prolongation.

In the present study, the ACE genotype was not significantly associated with QTd at baseline. We also found there was no difference in the baseline clinical characteristics among the different ACE genotypes. The possible explanation for this observation is that prolongation of QTd and its association with the ACE genotype are frequently observed in a diseased heart with LV dilatation, hypertrophy, or infarction. All of the present subjects underwent thorough examination and were free of organic heart disease. The lack of an association between ACE genotype and QTd in healthy subjects who are free from cardiovascular diseases same findings was also reported by Jeron et al! 4 Another explanation is that our ascertainment scheme only allowed active, independent elderly people to be eligible for enrollment. Therefore, if the $\mathrm{D}$ allele is a deleterious factor, we would overascertain healthy D-allele carriers at baseline. In fact, there are several reports that the $\mathrm{D}$ allele is related to cardiovascular diseases. ${ }^{10-14}$ Therefore, our present study results showed that the D-allele carriers had a larger increase of dispersion, but without longer QT variables at baseline. The other possible explanation is the small sample size.

Regarding the AGT M235T polymorphism, although it has been reported to be associated with plasma AGT, hypertension, cardiac hypertrophy and coronary artery disease, ${ }^{15,22}$ limited reports have discussed its relationship with arrhythmia ${ }^{34}$ Recently, Raizada et al reported no association of AGT M235T polymorphism with QT parameters among patients with end stage renal disease, based on a cross-sectional study 35 Although the $\mathrm{T}$ allele has been reported to be associated with cardiac structural change and atrial arrhythmia in Chinese, we did not find a link between QT parameters and AGT M235T polymorphism. In addition, the effect of AGT M235T polymorphism on cardiac AGT concentration is unclear, despite its association with plasma AGT levels. The lack of association in present study may be attributed to the small sample size or short duration of follow-up. For the TDR-related parameters, investigations of other genotypes, such as the KCNQ1 polymorphism suggested by Ozawa et al, may further clarify the genetic effect of these parameters in this longitudinal cohort?

\section{Study Limitations}

There are 3 limitations in this study. First, 87 (45\%) patients were lost to follow-up in the overall cohort. However, we found that there was no statistical significance in the baseline characteristics, including age, sex, height, weight, BMI, LVMI, systolic and diastolic blood pressures, between the those who did or did not complete the 4-year follow-up. Because these 87 subjects were excluded from our analysis at the beginning, our results should not be biased by the loss to follow-up. Second, the QT variables are influenced by many factors, such as electrolytes and autonomic tone. Because potassium level was not measured, we cannot discuss its influence on the QT variables. In this study all participants had $8 \mathrm{~h}$ of fasting before blood sam- 
pling between $08.00-09.00 \mathrm{~h}$ and normal renal function, which might reduce the fluctuation of potassium levels. Physical training could improve QTcd, possibly by improving the autonomic nerve system 36 Our elderly subjects had only light daily activity and $10 \mathrm{~min}$ rest before all examinations, which would lower the influence of autonomic tone change on the QT variables. Third, we had a relatively small sample size and only studied 2 genes, which may not provide enough power to investigate genes with minor effects. The limited sample size also restricted our explore of any gene-gene interactions. Although our findings are potentially intriguing, the result needs to be replicated for confirmation.

In conclusion, this longitudinal study disclosed an association between ACE I/D polymorphism and the magnitude of QT and QTcd prolongation in an elderly Chinese cohort. However, the AGT M235T polymorphism did not have a significant association with the phenotypes of interest. Further studies to replicate our results are mandatory.

\section{Acknowledgment}

This study was supported by National Health Research Institutes (NHRI-EX93-8903PL) from the Department of Health, Executive Yuan, Taiwan, ROC.

\section{References}

1. Fleg JL, Kennedy HL. Cardiac arrhythmias in a healthy elderly population: Detection by 24-hour ambulatory electrocardiography. Chest 1982; 81: $302-307$

2. Reardon M, Malik M. QT interval change with age in an overtly healthy older population. Clin Cardiol 1996; 19: 949-952.

3. de Bruyne MC, Hoes AW, Kors JA, Hofman A, van Bemmel JH, Grobbee DE. QTc dispersion predicts cardiac mortality in the elderly: The Rotterdam study. Circulation 1998; 97: 467-472.

4. Okin PM, Devereux RB, Howard BV, Fabsitz RR, Lee ET, Welty TK. Assessment of QT interval and QT dispersion for prediction of all-cause and cardiovascular mortality in American Indians: The Strong Heart Study. Circulation 2000; 101: 61-66.

5. Aiba T, Shimizu W, Inagaki M, Hidaka I, Tatewaki T, Sunagawa K. Transmural heterogeneity of the action potential configuration in the feline left ventricle. Circ J 2003; 67: 449-454.

6. Shimizu W, Antzelevitch C. Cellular basis for the ECG features of the LQT1 form of the long-QT syndrome: Effects of beta-adrenergic agonists and antagonists and sodium channel blockers on transmural dispersion of repolarization and torsade de pointes. Circulation 1998; 98: 2314-2322.

7. Ozawa T, Ito M, Tamaki S, Yao T, Ashihara T, Kita Y, et al. Gender and age effects on ventricular repolarization abnormality in Japanese general carriers of a G643S common single nucleotide polymorphism for the KCNQ1 gene. Circ J 2006; 70: 645-650.

8. Rigat B, Hubert C, Alhenc-Gelas F, Cambien F, Corvol P, Soubrier F. An insertion/deletion polymorphism in the angiotensin I-converting enzyme gene accounting for half the variance of serum enzyme levels. J Clin Invest 1990; 86: 1343-1346.

9. Danser AH, Schalekamp MA, Bax WA, van den Brink AM, Saxena $\mathrm{PR}$, Riegger GA, et al. Angiotensin-converting enzyme in the human heart: Effect of the deletion/insertion polymorphism. Circulation 1995; 92: 1387-1388.

10. Samani NJ, Thompson JR, O’Toole L, Channer K, Woods KL. A metaanalysis of the association of the deletion allele of the angiotensinconverting enzyme gene with myocardial infarction. Circulation 1996; 94: 708-712.

11. Marian AJ, Yu Q, Workman R, Greve G, Roberts R. Angiotensin converting enzyme polymorphism in hypertrophic cardiomyopathy and sudden cardiac death. Lancet 1993; 342: 1073-1075.

12. Anvari A, Turel Z, Schmidt A, Yilmaz N, Mayer G, Huber K, et al. Angiotensin-converting enzyme and angiotensin II receptor 1 polymorphism in coronary disease and malignant ventricular arrhythmias. Cardiovasc Res 1999; 43: 879-883.

13. Takahashi T, Ueno H, Yasumoto K, Kagitani S, Tomoda F, Inoue H, et al. Angiotensin-converting enzyme-gene polymorphism is associated with collagen I synthesis and QT dispersion in essential hyper- tension. J Hypertens 2003; 21: 985-991.

14. Jeron A, Hengstenberg C, Engel S, Lowel H, Riegger GA, Schunkert $\mathrm{H}$, et al. The D-allele of the ACE polymorphism is related to increased QT dispersion in 609 patients after myocardial infarction. Eur Heart J 2001; 22: 663-668.

15. Winkelmann BR, Russ AP, Nauck M, Klein B, Bohm BO, Maier V, et al. Angiotensinogen M235T polymorphism is associated with plasma angiotensinogen and cardiovascular disease. Am Heart $J$ 1999; 137: 698-705.

16. Young RP, Sanderson JE, Tomlinson B, Woo KS, Critchley JA. Angiotensin converting enzyme insertion-deletion polymorphism in Chinese. J Hypertens 1995; 13: 1479-1480.

17. Rotimi C, Cooper R, Ogunbiyi O, Morrison L, Ladipo M, Tewksbury $\mathrm{D}$, et al. Hypertension, serum angiotensinogen, and molecular variants of the angiotensinogen gene among Nigerians. Circulation 1997; 95: 2348-2350.

18. Malik M, Batchvarov VN. Measurement, interpretation and clinical potential of QT dispersion. J Am Coll Cardiol 2000; 36: 1749-1766.

19. Haraguchi Y, Yoshinaga M, Sarantuya J, Shimago A, Nishi J, Kono $\mathrm{Y}$, et al. Interval representative of transmural dispersion of repolarization in children and young adolescents with congenital long QT syndrome. Circ J 2005; 69: 78-82.

20. Devereux RB, Reichek N. Echocardiographic determination of left ventricular mass in man: Anatomic validation of the method. Circulation 1977; 55: 613-618.

21. Hsieh MC, Lin SR, Hsieh TJ, Hsu CH, Chen HC, Shin SJ, et al. Increased frequency of angiotensin-converting enzyme DD genotype in patients with type 2 diabetes in Taiwan. Nephrol Dial Transplant 2000; 15: $1008-1013$.

22. Wang AY, Chan JC, Wang M, Poon E, Lui SF, Li PK, et al. Cardiac hypertrophy and remodeling in relation to ACE and angiotensinogen genes genotypes in Chinese dialysis patients. Kidney Int 2003; 63: 1899-1907.

23. Su HM, Chiu HC, Lin TH, Voon WC, Liu HW, Lai WT, et al. Longitudinal study of the aging trends in QT interval and dispersion in healthy elderly subjects. Age Ageing 2006; 35: 636-638.

24. Savelieva I, Camm AJ, Malik M. QT dispersion should be adjusted for age: Observations from 1096 normal subjects (abstract). J Am Coll Cardiol 1993; 33(Suppl A): 129A.

25. Zhang N, Ho TF, Yip WCL. QT dispersion in healthy Chinese children and adolescents. Ann Noninvas Electrocardiol 1999; 4: $281-$ 285.

26. Macfarlane PW, McLaughlin SC, Rodger C. Influence of lead selection and population on automated measurement of QT dispersion. Circulation 1998; 98: 2160-2167.

27. Tutar H, Öcal B, Imamoglu A, Atalay S. Dispersion of QT and QTc interval in healthy children, and effects of sinus arrhythmia on QT dispersion. Heart 1998; 80: 77-79.

28. Higham PD, Furniss SS, Campbell RWF. QT dispersion and components of the QT interval in ischemia and infarction. Br Heart J 1995; 73: $32-36$.

29. Oikarinen L, Nieminen MS, Viitasalo M, Toivonen L, Wachtell K, Papademetriou V, et al. Relation of QT interval and QT dispersion to echocardiographic left ventricular hypertrophy and geometric pattern in hypertensive patients: The LIFE study (The Losartan Intervention For Endpoint Reduction) J Hypertens 2001; 19: 1883-1891.

30. Burlew BS. Diastolic dysfunction in the elderly: The interstitial issue. Am J Geriatr Cardiol 2004; 13: 29-38.

31. Schunkert H, Hense HW, Holmer SR, Stender M, Perz S, Keil U, et al. Association between a deletion polymorphism of the angiotensinconverting-enzyme gene and left ventricular hypertrophy. $N$ Engl J Med 1994; 330: 1634-1638.

32. Capasso JM, Malhotra A, Remily RM, Scheuer J, Sonnenblick EH. Effects of age on mechanical and electrical performance of rat myocardium. Am J Physiol 1983; 245: H72-H81.

33. Kuznetsova T, Staessen JA, Thijs L, Kunath C, Olszanecka A, Ryabikov A, et al. Left ventricular mass in relation to genetic variation in angiotensin II receptors, renin system genes, and sodium excretion. Circulation 2004; 110: 2644-2650.

34. Tsai CT, Lai LP, Lin JL, Chiang FT, Hwang JJ, Ritchie MD, et al. Renin-angiotensin system gene polymorphisms and atrial fibrillation. Circulation 2004; 109: 1640-1646.

35. Raizada V, Skipper B, Luo W, Garza L, Hines CW, Harford AA, et al. Renin-angiotensin polymorphisms and QTc interval prolongation in end-stage renal disease. Kidney Int 2005; 68: 1186-1189.

36. Nishiyama Y, Maeda H, Tanaka M, Hirano K, Koga Y. Effect of physical training on corrected QT dispersion in patients with nonischemic heart failure. Circ J 2004; 68: 946-949. 